\title{
COMPARATIVE ANALYSIS OF SINGULARITIES OF 3-PRS AND 3- PRS PARALLEL MANIPULATORS USING GRASSMANN-CAYLEY ALGEBRA
}

\author{
Luc Djimon Clément AKONDE
}

\begin{abstract}
Ph-D Student, State Key Laboratory of Digital Manufacturing Equipment and Technology, Mechanical school of
\end{abstract} Science \& Engineering, Huazhong University of Science and Technology, Wuhan, China, goering20@yahoo.fr

\begin{abstract}
Grassmann-Cayley Algebra (GCA) is a symbolic approach to Plücker coordinates lines. It is used to write expressions for geometric incidences in projective geometry.GCA gives a useful way to analyze mechanism singularity without algebraic coordinate expression. Since it has great powerful tools for coordinate's free representation, geometric interpretation and singularity analyzing in real time computing, this method is implemented in the present research. The goal of this study is finding the singularities conditions of 3-PRS Parallel Manipulators (PMs) based on GCA when the position of the actuators is firstly on prismatic joints and secondly on revolute joints. Geometric method of reciprocal screw is used to formulate the Global Wrench System (GWS) of the PMs. GWS represents the determinant of Jacobian matrix which rows are Plücker coordinate lines. The degeneracy analysis of determinant of this matrix based on the dependency notion of Plücker coordinate lines is performed in coordinate free expressions and provides double and single singularities conditions, respectively for3-PRS and 3-PRS PMs, which contains all generals and specials cases. The keys elements of transition between the rows of Jacobian matrix and singularity condition are the introduction of Plücker lines, superbrackets, join and meet operators. The vanishing points of these superbrackets decomposition are analyzed to describe the singularity conditions associated with different motions. The results indicate that for the 3-PRS and 3-PRS PMs, a common singularity occurs when the three constraints forces of the mobile platform lie on the common plane and intersect at a unique point. It is suggested that both position and orientation of the actuator have to be integrated in the conceptual design stage in order to optimize the rigidity frame of the mechanism.
\end{abstract}

Key Words: Singularity, 3-PRS Parallel Manipulators, Grassmann-Cayley Algebra, Projective space, Wrench graph.

\section{INTRODUCTION}

Robot singularity has always been a classical problem for mechanical designers. Singularity is commonly referred to a position in the robot's workspace where the joints no longer work as independent controlling variables. The level of difficulty involved in computing the determinant $\operatorname{det}(J)$ of the Jacobian matrix $(J)$ associated with the Plücker coordinate lines, is closely related to the complexity of the robot mechanism. The more complicate its architecture, the more difficult $\operatorname{det}(J)$ is to be calculated. Parallel singularity occurs when the robot's mobile platform gains extra degree of freedom ( $d o f$ ) and becomes uncontrollable. To overcome this problem, several methods have proposed over the past years. Among these methods, the GrassmannCayley Algebra $(G C A)$ is probably one of the most appealing since it provides sufficient tools to properly solve the abovementioned problem due to its merits. First of all, $G C A$ is suitable for analyzing the rigidity of the framework of the architecture and for scene analysis. It's a bridge which allows interaction between symbolic and numerical computation [1-11].Secondly, it has a powerful coordinate free representation which means $G C A$ has a high capability to work and make representation shortly in coordinate free manners. A reciprocal translation between geometric entities and algebraic expressions can be obtained [1-17].The third motivation is its geometrical generic interpretation: $G C A$ has a suitable language for interpreting geometrically singularity of parallel manipulators $(P M s)$.The tools such as superbrackets have ability to analysis the constraints applied on the moving platform and compare manipulators at the conceptual design stage $[1-5 ; 10-15 ; 18-19]$. Another interesting feature of $G C A$ is that it has the ability on real time computing. The computational time needed to calculate the singularity equation in $G C A$ is fairly short and low cost. The solution provided by $G C A$ is a single condition that contains all of the special cases in which the robot is singular $[1-4 ; 9-11 ; 15]$. To prevent the parallel singularity, and the robot's performance deterioration, it is necessary to obtain, firstly the $J$ related to its instantaneous motion and secondly calculate $\operatorname{det}(J)$ degeneracy.

Because 3-PRS (prismatic, revolute and spherical) robots have a wide industrial application, they have been intensively investigated over the past few years using different approaches [56-58]. For example, Tsai et al [20] in order to detect the undesirable configurations of 3-PRS PMs compared both Bezout's elimination method and optimization techniques while $\mathrm{Li}$ and $\mathrm{Xu}$ [21]used screw 
theory and its reciprocal to represent the mobility of 3-PRS PMs and investigated algebraically the singularity condition after a numerical calculus of $J$.Other approaches of solving singularity of PMs included the application of the algebraic properties of Lie group [22], kinematics models [23], nonpure rotational and non-pure translational $J$ [24], numerical analysis[25], analytical solution based on MATLAB programming [26], analytical expression in singularity locus of general Stewart-Gough PMs [27], discretization of the $J$ [28], etc...

Some challenges are still encountered in designing 3-PRS PMs. Even if their singular positions could be successfully detected by software simulation [29], parallel robot singularity and its associated motion still are an interesting research field for robot designers due to the complex nature of the topic. Joshi and Tsai [30] used screw theory and it reciprocal to present the Jacobian matrice of 3-UPU and 3RPS PMs which provides information about both architecture and constraint singularities while X. Ding et al [31] proposed recently a novel deployable prism mechanism. The singularity motion associated to his parallel manipulator was studied by screw theory method and provided two singularities in the whole mechanism when it was deploying.

It has been shown that singularity analysis of parallel manipulator cannot be performed without manipulating the $J$ associated with the instantaneous motion of the moving platform and/or the actuated joint of the manipulator [3234]. J.P Merlet [35] confirmed that calculating the determinant is only the first stage, the second stage is finding the poses that cancel the determinant is the most difficult.

In this paper we focus on the poses that cancel the determinant using $G C A$. This method allows us to analyze the singularity in coordinate-free manners and make a geometric interpretation for the vanishing point of the superbrackets decomposition. The superbrackets are alternative manners to calculate $\operatorname{det}(J)$.

In this research, the comparative analysis of 3- $\underline{P} R S \quad P M S$ and 3-P $\underline{R} S$ PMs based on the degeneracy of each Global Wrench System $(G W S)$ using the dependency of Plücker coordinate lines is performed in coordinate free expression to obtain respectively a double and a single singularity conditions including all special cases. The work is organized as follows: Sect. 2 recalls some mathematical background of $G C A$ used in projective space such as a concept of lines, reciprocal screw theory, and $G W S$ before join -meet operators. In Sect.3, the 3-PRS PMs and the adopted representation of the mechanism are described. Geometrically, $G W S$ which is similar to $J$ is determined by the reciprocal screw theory before the symbolic approach of Plücker coordinate lines in Sect.4 while in Sect.5, the singularity conditions and associated motions are discussed. .Finally the conclusion is given at Sect.6.

\section{MATHEMATICAL BACKGROUND AND FUNDAMENTAL CONCEPTS}

In this section we will give a brief overview of $G W S$ in projective space before a symbolic approach of Plücker coordinate lines in $G C A$. More details on $G C A$ and superbrackets decomposition should refer to $[4,7,16,19$, 52-55].

\subsection{Projective Space}

The projective space refers to the affine space augmented by the points at infinity where any pair of parallel lines can be said to meet at the unique point at infinity on the plane $\Pi_{\infty}$. Toms [36] recalled three important axioms from projective geometry: firstly for any given two distinct points in a plane, there is a unique line that lies on both of them, secondly for any given two distinct lines in a plane, there is a unique point that lies on both of them and at last all algebraic representation of projective geometry must be homogeneous. In projective space any point (line) has four (six) homogeneous coordinates and each line is called Plücker line which can be extended to coordinates for the screws and the duality twist-wrenches in application for kinematics of robot manipulators[17,37-38]. Screw theory has been an important tool in mechanics of robotics [39-42]. A finite line, $L$, is represented by its Plücker coordinates vector $F$ :

$$
F=(s ; r \times s)
$$

where $s$ is the line direction, $(r \times s)$ represents the moment of $L$ with respect to the origin and $r$ is the position vector of any point on $L$. An instantaneous screw axis is a Plücker coordinate vector line with it associated pitch in a given position, then the screw axis of PMs is described by:

$$
\hat{\$}=\left[\mathrm{s} ;\left(\mathrm{s}_{0} \times s+h s\right]^{T}\right.
$$

with, $s$ the unit vector along the screw axis, $s_{0}$ the position vector of a point on the screw axis with respect to a reference frame, $h$ the pitch of the screw. A zero pitch screw $\hat{\$}_{0}$ and an infinite pitch screw $\hat{\$}_{\infty}$ can be respectively identified with a Plücker coordinate vectors of a finite line and a line at infinity:

$$
\begin{gathered}
\hat{\$}_{0}=\left[s ;\left(s_{0} \times s\right)\right]^{T} \\
\hat{\$}_{\infty}=[0 ; s]^{T}
\end{gathered}
$$

A twist is a screw representing the instantaneous motion (linear and angular velocity) of a rigid body. A body instantaneously undergoing a pure rotation about an axis $l$ is a twist of zero pitch $\hat{\$}_{0}$. A body instantaneously undergoing a pure translation along an axis $l$ is a twist of infinite pitch $\hat{\$}_{\infty}$. A wrench is a screw representing a combination of a force and a couple acting on a rigid body. A body subject to a pure force along the axis $l$ is a wrench of zero pitch 
screw $\hat{\$}_{0}$. A body subject to a pure couple is a wrench of infinite pitch screw $\hat{\$}_{\infty}$.

\subsection{Geometrical Method for Reciprocal Screw}

\section{Theory}

Since $n$ number of independents kinematic chains form $(n)$ screw space which is composed of $(n)$ system screws, all screws which are reciprocal to a $(n)$ system screws form a $(6-n)$ system [14, 30, 44-51]. Two zero pitch screws $\hat{\$}_{0}$ and $\hat{\$}_{0}^{\prime}$ are reciprocal to each other if and only if their axes are coplanar. A zero pitch screw $\hat{\$}_{0}$ is reciprocal to an infinite pitch screw $\hat{\$}_{\infty}$ if only if their directions are orthogonal to each other. Two infinite pitch screws $\hat{\$}_{\infty}$ and $\hat{\$}_{\infty}^{\prime}$ are always reciprocal to each other. [30, 46-49].

\subsection{Global Wrench System (GWS)}

GWS is a platform wrench system which is obtained by the combination of the actuation wrench system $\left(W_{n}\right)$ and the constraint wrench system $\left(F_{i}\right)$ [18]. The constraint wrench system of $l_{i}$ is identified as a reciprocal screw system to all the joint screws of $l_{i}$.Its depends of the architecture of the manipulator and defines how the mobile platform is forced by $l_{i}$. while the actuation wrench system of $l_{i}$ is depend on the operating mode and define how the actuators act on the $d o f$ of the effectors. It is obtained my locking the actuators of $l_{i}$ before determining all reciprocal screw to the joint screws except that of the actuated $\operatorname{lin} k_{n}$ of $l_{i}$. The GWS is described as:

$$
G W S=\left(W_{n}, F_{i}\right)=\left(\hat{\$}_{a}^{1}, \ldots, \hat{\$}_{a}^{n}, \hat{\$}_{c}^{1}, \ldots, \hat{\$}_{c}^{6-n}\right)
$$

$$
\text { with } W_{n}=\underbrace{\hat{\$}_{a}^{1}, \ldots, \hat{\$}_{a}^{n}}_{(n) \text { system-actuated }} \text { and } F_{i}=\underbrace{\hat{\$}_{c}^{1}, \ldots, \hat{\$}_{c}^{6-n}}_{(6-n) \text { system-constraint }}
$$

The $\operatorname{det}(J)$ is written in the $G W S$ form as:

$$
\operatorname{det}(J)=[G W S]=[\underbrace{W_{1}, \ldots, W_{n}}_{(\text {n) system }}, \underbrace{F_{1}, \ldots, F_{i}}_{(6-n) \text { system }}]
$$

\subsection{Grassmann-Cayley Algebra based on Plücker} coordinates lines

Let $V^{n}$ be $n$-dimensional vector space over the field $R$, $W$ be $k$-dimensional subspace of $V^{n}$ with $\left\{w_{1}, w_{2}, \ldots, w_{k}\right\}$ a basis of $W$ and $P$ a Plücker coordinate vector of $W$. A symbolic level of $P$ without specific coordinate is also a Plücker coordinate vector $P$ in the $\partial_{n}^{k}$-dimensional vector space $V^{k}$ and is written as [5]:

$$
P=w_{1} \vee w_{2} \vee \ldots \vee w_{k}=w_{1} w_{2} \ldots w_{k}
$$

In coordinate free expression $P$ is called an extensor of step $k$. Let be $S$ a set of finites points $\left\{w_{1}, w_{2}, \ldots, w_{k}\right\}$ defined now in the $(k-1)$ dimensional projective space $P$ over the field $R$ where $w_{i}=\left(x_{1, i}, x_{2, i}, \ldots, x_{k, i}\right)^{T}$. Each $w_{i}$ is represented by it homogeneous coordinate form $k$-tuple with $1 \leq i \leq k$. The bracket of these vectors is defined as the determinant of matrix having $w_{i}$ as its columns and described as:

$$
\left[w_{1}, w_{2}, \ldots, w_{k}\right]=\operatorname{det}\left|\begin{array}{cccc}
x_{1,1} & x_{1,2} & \cdots & x_{1, k} \\
\vdots & \vdots & \cdots & \vdots \\
x_{k, 1} & x_{k, 2} & \cdots & x_{k, k}
\end{array}\right|
$$

This determinant in $G C A$ language represents the symbolic approach of Plücker coordinate lines which is similar to the superbrackets and is used to analyze the singularity without algebraic coordinates.

\subsection{Join and Meet Operators}

The symbols $\vee$ and $\wedge$ represent respectively the operators join and meet [1-3]. The join of two or more extensors represent the operation of (union) joining the associated vector subspaces while meet is for the intersection of subspaces. These tools are useful in the interpretation of singularity conditions Robot Manipulators. Instantaneously the wrench space of parallel combination of motion is the sum of the wrench spaces of the composing constraints. Then in $G C A$ language for kinematics chains the wrench space of the parallel connection of kinematic chains is the support of the join of the extensors that represent the wrench spaces of the chains, provided that their wrench extensors are linearly independent [8]. In consequence, the system Eq. (8) is linearly dependent when:

$$
w_{1} \vee w_{2} \vee \ldots \vee w_{k}=\left[w_{1}, w_{2}, \ldots, w_{k}\right]=0
$$

\section{DESCRIPTION AND ADOPTED}

\section{REPRESENTATIONS OF 3-PRS PARALLEL MANIPULATORS}

\subsection{Description of 3-PRS Parallel Manipulators}

The chosen architecture (Fig-1) is similar to Tsai's [20]. 


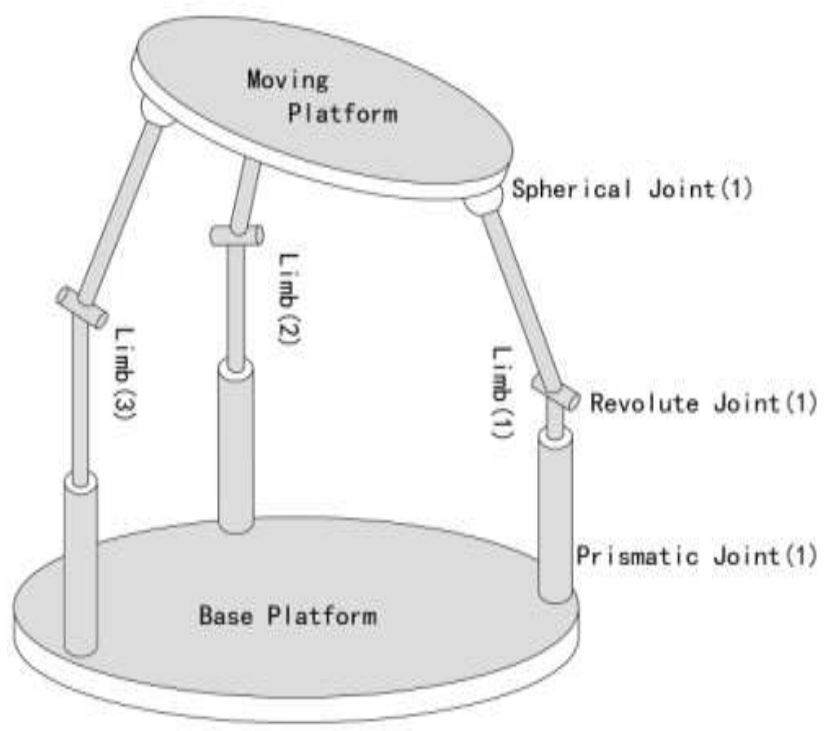

Fig -1: 3-PRS Parallel Manipulators designed by Tsai

The moving platform $(M)$ centered on $O^{\prime}\left(x^{\prime}, y^{\prime}, z^{\prime}\right)$ is connected to a fixe base $(F)$ centered on $O(x, y, z)$ by three fully-parallel $\operatorname{limbs} l_{i}$.Each $\operatorname{limb} l_{i}$ consists of a prismatic joint $(P)$ fixed on $(F)$ at point $P_{i}$,followed by a revolute joint $(R)$ at a point $\left(R_{i}\right)$ about which rotates a fixed-length link and the connecting link to the end-effector is a spherical joint $(S)$ at point $S_{i}$ on $(M)$ (see Fig-2).

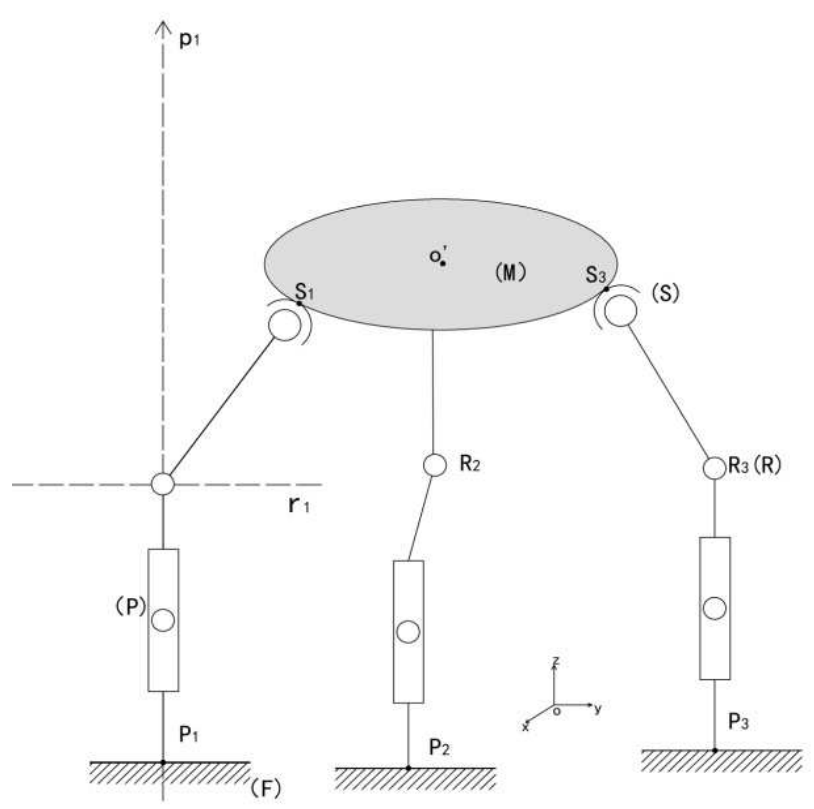

Fig -2: Mechanism architecture

Each independent $l_{i}$ has five degrees of freedom express as one translation -one rotation- and one spherical which is consist of three intersecting and non-coplanar rotations joints at $S_{i}\left(u_{i}, v_{i}, w_{i}\right)$. For example $l_{1}$ has a translational point $P_{1}$ fixed on $(F)$ followed by a rotated point $R_{1}$ which is connected to the link of the end effector by a spherical point $S_{1}\left(u_{1}, v_{1}, w_{1}\right)$ on $(M)$ where $S_{1} u_{1}, S v_{1}$ and $S_{1} w_{1}$ are respectively three intersecting noncoplanar revolute joint axis at $S_{1}$ (see Fig-3).

\subsection{Adopted Representations of 3-PRS Parallel Manipulators}

(a) The input of the mechanism adopted in this paper consists of two variable positions of actuated joint. Firstly the prismatic joints are actuated and all other joints are passive. Secondly the revolute joints are actuated and all other joints are passive. The order of each limb is respectively represented by the letter $i=1,2,3$.

(b) The axis $P_{i}$ of prismatic joint and the following axis $r_{i}$ of revolute joints are orthogonal.

(c) Let be $\alpha_{i}$ the plane formed by the spherical joint center $S_{i}$ and the joint axis $r_{i}$

(d) Let be $\beta_{i}$ the plane which contains the spherical joint

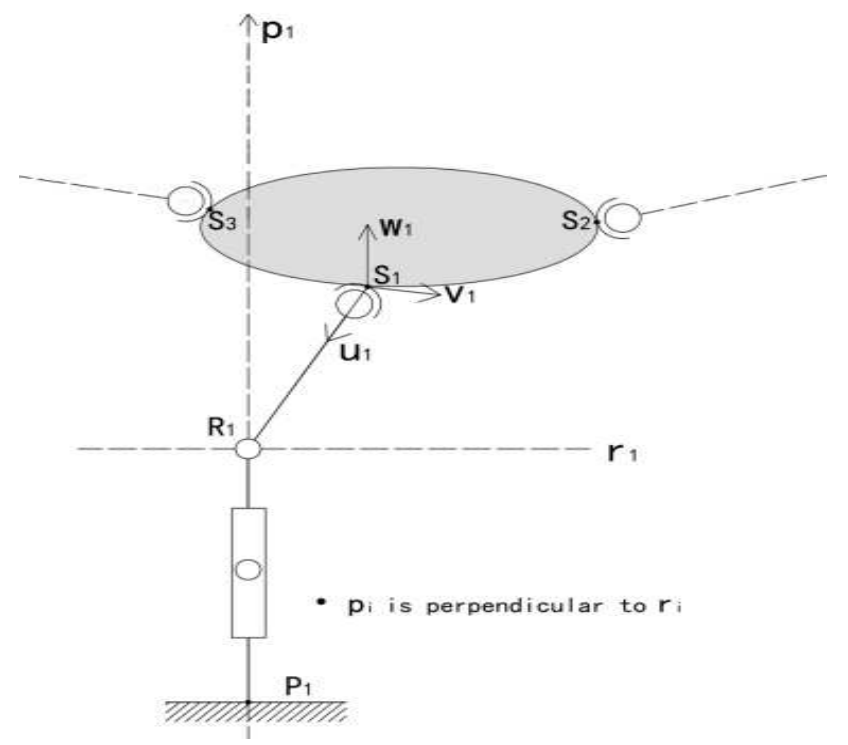

Fig-3: Architecture of $l_{i}$

center $S_{i}$ and perpendicular to the prismatic joint $p_{i}$. Consequently:

(e) $\alpha_{i}$ and $\beta_{i}$ intersect each other at a line $F_{i}$.

(f) Lines $a b$, ef and $i j$ represent respectively $F_{1}, F_{2}$ and $F_{3}$ while $c d, g h$ and $k l$ represent respectively $r_{1}, r_{2}$ and $r_{3}$ thus each $F_{i}$ parallel to $r_{i}$ (see Fig-4).

The dof of a 3-PRS PMs is expressed as[21] : 


$$
d o f=\lambda(n-g-1)+\sum_{i=1}^{g} X_{i}
$$

$d o f=6(8-9-1)+(5+5+5)=3$ where $n, g$ and $X_{i}$ are respectively the number of bodies, joints and degree of freedom of the $i-t h$ joint of the mechanism.

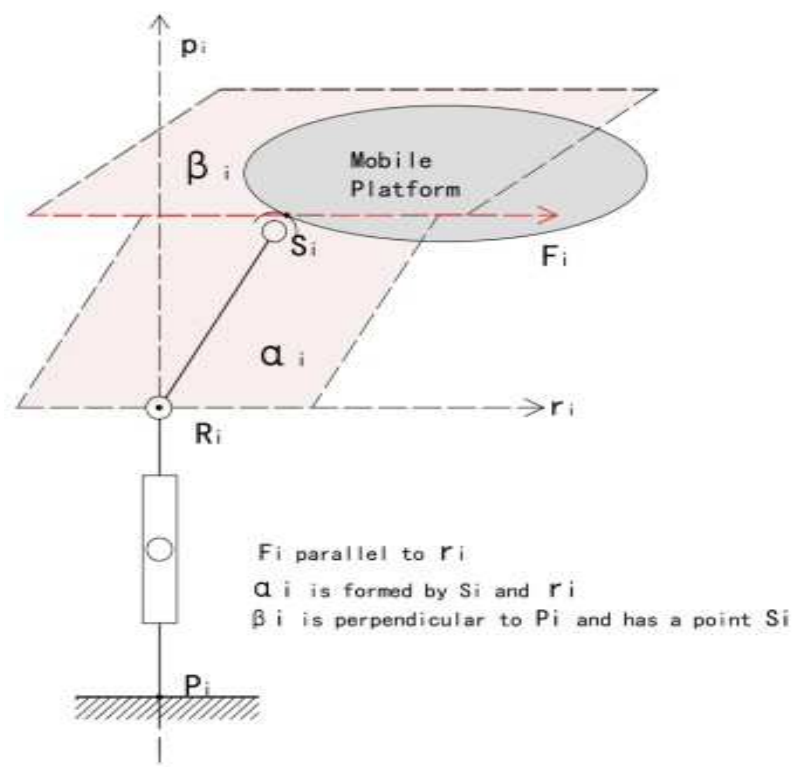

Fig-4: Actuated and constraint wrench system

Since each limb $l_{i}$ of a 3-PRS PMs consist of five serial kinematic chains, a twist $T^{i}$ of each $\operatorname{limb} l_{i}$ form 5 -system [30, 46-51], see Fig-3 and Eq. (12). Instantaneously the composition of these five serial twists corresponds to their simple addition in the projective space [8].

$$
T^{i}=\left[\left(\hat{\$}_{p i}\right)+\left(\hat{\$}_{r i}\right)+\left(\hat{\$}_{u i}\right)+\left(\hat{\$}_{v i}\right)+\left(\hat{\$}_{w i}\right)\right]
$$

With

$\hat{\$}_{p i}=\left[0, p_{i}\right]^{T} ; \hat{\$}_{r i}=\left[r_{i},\left(R_{i} \times r_{i}\right)\right] ; \hat{\$}_{u i}=\left[u_{i},\left(S_{i} \times u_{i}\right)\right]$;

$\hat{\$}_{v i}=\left[v_{i},\left(S_{i} \times v_{i}\right)\right] ; \hat{\$}_{w i}=\left[w_{i},\left(S_{i} \times w_{i}\right)\right]$

where $p_{i}, r_{i}, u_{i}=S_{i} u_{i}, v_{i}=S_{i} v_{i}$ and $w_{i}=S_{i} w_{i}$ are respectively the unit vectors along the prismatic, revolute and the three intersecting non-coplanar revolute joints axis of the spherical joint for the $i-t h$ limb (see Fig-3).

\section{GWS AND SYMBOLIC APPROACH OF PLUCKER LINES OF 3-PRS PMS}

\subsection{Constraint Wrench System and Actuated Wrench System of 3-PRS PMs}

Constraint wrench system $F_{i}$ :each limb $l_{i}$ form a (5)system twist $T^{i}$, the reciprocal constraint wrench system to all (5) system twist $T^{i}$ form a (1)ystem constraint wrench of zero pitch [30,44-51]. The constraint wrench system $F_{i}$ of each limb $l_{i}$ is defined as a line force passing through the center of the spherical joint $S_{i}$ along the direction parallel to the revolute joint $r_{i}$ [43] (See Fig-4)

$$
F_{i}=\left[r_{i},\left(\mathrm{~S}_{i} \times r_{i}\right)\right]
$$

Actuated wrench system.

When the actuated prismatic joint of $l_{i}$ is locked, only $4-$ system twist is valid. Consequently each prismatic actuated wrench, $W_{i}$, of each $l_{i}$ form 2 -system wrench and only reciprocal to all passive 4-system twist $\hat{\$}_{r i}, \hat{\$}_{u i}, \hat{\$}_{v i}$ and $\hat{\$}_{w i}$. Then all reciprocal screws lie on a plane $\alpha_{i}$ (see Fig-4) [43].

When the actuated revolute joint of $l_{i}$ is locked, only 4 - system twist is valid. Consequently each revolute actuated wrench, $W_{i}^{\prime}$, of each $l_{i}$ form $2-$ system wrench and only reciprocal to all passive 4-system twist $\hat{\$}_{p i}, \hat{\$}_{u i}, \hat{\$}_{v i}$ and $\hat{\$}_{w i}$. Then all reciprocal screws lie on a plane $\beta_{i}$ (see Fig-4) [43].

\subsection{The Global Wrench System and Symbolic} Approach of Plücker Coordinate Lines of 3-PRS PMs

The GWS explains all characteristic efforts which influence the end-effector and defined as a join of actuated wrench and constraints wrench.

$1^{\text {st }}$ case : $3-P R S$

$$
\begin{aligned}
& \operatorname{det}\left(\mathrm{J}_{6 \times 6}\right)=G W S \\
& G W S=\left[\alpha_{1}, \alpha_{2}, \alpha_{3}, F_{1}, F_{2}, F_{3}\right] \text { with } \\
& F_{i} \in \alpha_{i}
\end{aligned}
$$

Since any plane can be defined by two different lines and any line is formed by two different points, and according to the adopted representation, the Eq. (13) can be rewrite as:

$$
G W S=[a b, c d, e f, g h, i j, k l]
$$

In GCA language, the symbolic approach of these six Plücker coordinates lines is described as:

$$
P=a b \vee c d \vee e f \vee g h \vee i j \vee k l
$$

$2^{\text {nd }}$ case $: 3-P \underline{R} S$

Similarly:

$$
\begin{aligned}
& \operatorname{det}\left(J_{6 \times 6}^{\prime}\right)=(G W S)^{\prime} \\
& (G W S)^{\prime}=\left[\beta_{1}, \beta_{2}, \beta_{3}, F_{1}, F_{2}, F_{3}\right] \text { with } \\
& F_{i} \in \beta_{i}
\end{aligned}
$$


Since a point which is not belonging to one line can form one plane with that line, the Eq. (16) can be rewrite as:

$$
(G W S)^{\prime}=[a b, b n, e f, f p, i j, j r]
$$

The symbolic approach of these six Plücker lines is:

$$
p^{\prime}=a b \vee b n \vee e f \vee f p \vee i j \vee j r
$$

\subsection{Wrench Graph of 3-PRS Parallel Mechanism}

The graphical representation of GWS is called wrench graph.

\section{$1^{\text {st }}$ case : See Fig-5.}

Each $F_{i}$ and $r_{i}$ are parallel, in projective geometry they intersect each over at infinity at a unique point. Then $b \equiv d, f \equiv h$ and $j \equiv l$ respectively for limbs $l_{1}, l_{2}$ and $l_{3}$.For notation convenience, let designed by the capital letters these points at infinity and rewrite Eq. (14) and Eq. (15)

$$
\begin{aligned}
& G W S=[a B, c B, e F, g F, i J, k J] \text { and } \\
& P=a B \vee c B \vee e F \vee g F \vee i J \vee k J
\end{aligned}
$$

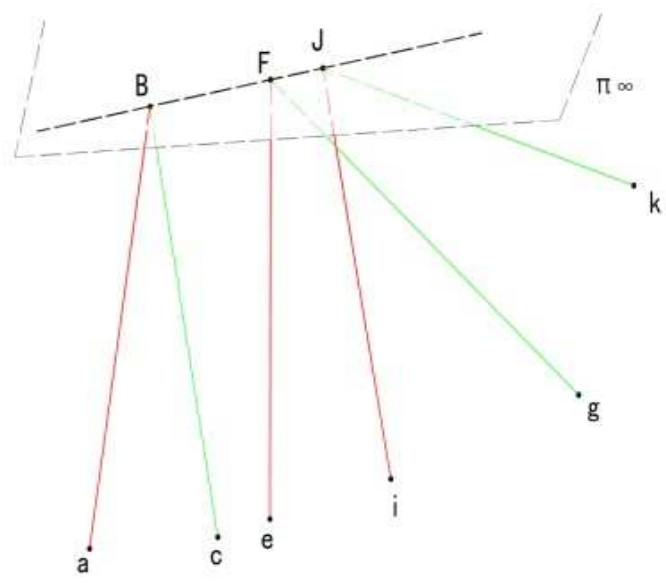

Fig-5: Wrench graph of 3-PRS PMs

$2^{\text {nd }}$ case : See Fig-6.

Any line can be represented in projective space by one finite point and one point at infinity, let choose one point at infinity on each of these six Plücker lines. Then Eq. (17) and Eq. (18) can be rewrite as:

$$
\begin{aligned}
& (G W S)^{\prime}=[A b, b N, E f, f P, I j, j R] \text { and } \\
& P^{\prime}=A b \vee b N \vee E f \vee f P \vee I j \vee j R
\end{aligned}
$$

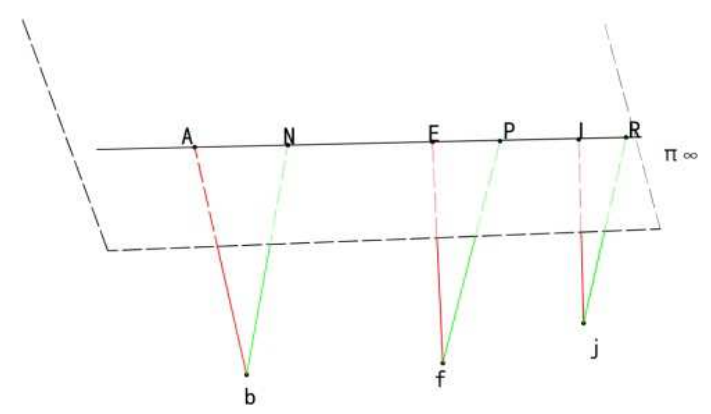

Fig-6: Wrench graph of 3-PRS PMs

\subsection{Superbrackets Decomposition of 3-PRS Parallel}

\section{Mechanism}

The superbrackets in Eq. (19) and Eq. (20) are generally developed into 24 combinations of linear monomials $[1,3]$. The Graphic User Interface (GUI) provided by Stéphane Caro et al [19] gives the reduced form of the superbrackets decomposition and the singularity conditions.

For 3-PRS PMs:

$$
\begin{aligned}
& {[a B, c B, \mathrm{eF}, \mathrm{gF}, \mathrm{iJ}, \mathrm{kJ}]=} \\
& +[\mathrm{aBce}][\mathrm{BgFJ}][\mathrm{FikJ}] \\
& -[\mathrm{aBcF}][\mathrm{BgFJ}][\mathrm{eikJ}] \\
& -[\mathrm{aBcg}][\mathrm{BeFJ}][\mathrm{FikJ}] \\
& +[\mathrm{aBcF}][\mathrm{BeFJ}][\text { gikJ }]
\end{aligned}
$$

For 3-PRS PMs:

$$
\begin{aligned}
& {[A b, b N, E f, f P, I j, j R]=} \\
& -[A b N E][b f P j][f I j R] \\
& +[A b N f][\mathrm{bfPj}][E I j R] \\
& +[A b N f][b E f j][P I j R] \\
& -[A b N P][b E f j][f I j R]
\end{aligned}
$$

\section{SINGULARITY CONDITIONS ASSOCIATED MOTIONS}

AND

Kinematically singularities arise when the moving platform becomes uncontrollable and actuators are useless. Algebraically the determinant of Jacobian matrix becomes zero. Geometrically in GCA language the six Plücker coordinate lines are linearly dependent. The superbracket which represents the determinant of the Jacobian matrice vanish.The analysis of the vanishing points of the superbracket is the singularity conditions. The GUI provides the solutions [19]

$$
\begin{aligned}
& 1^{\text {st }} \text { case }:[a B, c B, e F, g F, i J, k J]=0 \text { when } \\
& {[x B F J]=0 \text { or } a B c \wedge i k J \wedge e F} \\
& 2^{\text {nd }} \text { case }:[\mathrm{Ab}, \mathrm{bN}, \mathrm{Ef}, \mathrm{fP}, \mathrm{Ij}, \mathrm{jR}]=0 \text { when } \\
& A b N \wedge b f j \wedge I j R \wedge E P f
\end{aligned}
$$




\subsection{Interpretation of the Associated Motion}

According to our chosen mechanism and adopted representation, each $\operatorname{limb} l_{i}$ is identified by $F_{i}$ and $W_{i}$ thus:

$1^{s t}$ case . $x=\{a, b, c, e, g, \mathrm{i}, k\} \quad$, in singular configuration: $[x B F J]=0$ or $a B c \wedge i k J \wedge e F$ If $[a B F J]=0$ then $a B \wedge F J$ means $F$ collinear to $a B$ or $J$ collinear to $a B$.Since $F$ and $J$ are respectively the unique intersecting point at infinity of parallel lines $e F, g F$ and $i J, k J$ then $F$ collinear to $a B$ or $J$ collinear to $a B$ means $a B$ intersect also $e F, g F, i J$ and $k J$ at $F$ as infinity point. Since two parallel lines intersect each over at a unique point at infinity it is obvious that $a B$ is parallel to $e F, g F, i J$ and $k J$.This means $F_{1}$ parallel to $F_{2} W_{2} F_{3} W_{3}$.Similarly if $[c B F J]=0$ with the same processes, $W_{1}$ Parallel to $F_{2}, W_{2}, F_{3}, W_{3}$.For $l_{1}$, the singularity occurs when $F_{1}$ or $W_{1}$ parallel to $F_{2}, F_{3}, \mathrm{~W}_{2}, \mathrm{~W}_{3}$.It is obvious to deduce for $l_{i}$.If $a B c \wedge i k J \wedge e F$ means the line $F_{2}$ crosses the intersection line of the two planes $\alpha_{1}$ and $\alpha_{3}$

For 3-PRS PMs, the singularity conditions are:

- The robot reach in singularity when the actuated force or the constraint force of any $l_{k}$ is parallel to either a constraint force or an actuated force of $l_{k^{\prime}}$ with $k \neq k^{\prime}$

- Singular motion arises when the common actuated force of $l_{1}$ and $l_{3}$ crosses the constraint force of $l_{2}$.

$2^{\text {nd }}$ case. The singular configuration arises when the four planes $\beta_{1}, \beta_{2}, \beta_{3}$ and $b f j$ intersect at a unique point.

For 3-PRS PMs the singularity condition is:

- The common actuated wrench of the three limbs crosses the plane formed by the three constraint forces at a point and then the robot manipulator is in a plane configuration.

Finally a comparative analysis provides that a common singularity condition occurs when the three constraints forces lie on a common plane and intersect at the particular point :the robot manipulator reaches in a plane configuration

\section{CONCLUSIONS}

This paper presented the singularity analysis of 3-PRS PMs with variable actuated joint using Grassmann-Cayley Algebra. We determined geometrically the GWS by reciprocal screws approach and used an alternative coordinate free method namely GCA to detect the singularity conditions. The graph of the GWS was obtained by introducing the notion of join and meet operator in projective space. An efficient method to investigate singular configuration from GWS was given by GCA. Based on this approach a double and a single singularity conditions respectively for 3-PRS and 3-PRS PMs were derived. These conditions contained all general and specific critical poses. The comparative analysis of these result suggested that both position and orientation of the actuator have to be integrated in the conceptual design stage in order to optimize the rigidity frame of the mechanism. It was deduced that a common singularity condition occurs when the three constraints forces lie on a common plane and intersect at the particular point where the robot manipulator reaches in a plane configuration.

Even though the current research did not cover all variety and complexity of singularity configuration of 3-PRS PMs, these results may motivated further research question, especially toward the singularity conditions of serial manipulator using the GCA approach since each limb $l_{i}$ of 3PRS PMs consists of serial kinematic chains. Elsewhere the powerful tools of GCA are also useful in robotics vision and image modeling.

\section{ACKNOWLEDGEMENT}

I'm grateful to Professor Stéphane Caro for his inspiring advice, to the editor and reviewers for their support.

\section{REFERENCES}

[1].Patricia Ben-Horin and Moshe Shoham. Singularity Analysis of a Class of Parallel Robots based on GrassmannCayley Algebra. Mechanism and Machine Theory, 41,no.8(2006),958-970.

[2].Patricia Ben-Horin and Moshe Shoham. Singularity Condition of Six-Degree-of-Freedom Three-Legged Parallel Robots Based on Grassmann-Cayley Algebra. IEEE Transactions on Robotics,22,no.4(2006),577-590.

[3].P.Ben-Horin ,M. Shoham. Application of Grassmann Cayley Algebra to Geometrical Interpretation of Parallel Robot Singularities. The International Journal of Robotics Research ,28,no.1(2009),127-141.

[4].Neil L. White. Grassmann-Cayley Algebra and Robotics Applications, in : Handbook of Geometric Computing. Applications in Pattern Recognition,Computer Vision, Neural computing, and Robotics,8,Springer-Verlag Berlin Heidelberg (2005), pp 629 -656.

[5] .Neil L. White .Grassmann-Cayley Algebra and Robotics. Journal of Intelligent and Robotic Systems, 11,(1994)91-107.

[6]. L. Fuchs. Algèbre de Grassmann-Cayley. XLIM-SIC, Université de Poitiers, France INRIA Sophia-Antipolis (2009)1-13.

[7].Neil L. White . A Tutorial on Grassmann-Cayley Algebra, in : Invariant Methods in Discrete and Computational Geometry ,Proceedings of the Curaçao Conference. Springer Netherlands(1994) pp 93-106.

[8].E. Staffetti, F.Thomas. Kinestatic Analysis of Serial and Parallel Robot Manipulators Using Grassman-Cayley Algebra, in: J.Lenarcic and M.M. Stanisic (eds.), Advances in robot Kinematics, Springer Netherlands (2000), pp17-27.

[9]. L. Fuchs ,L. Théry. A Formalization of GrassmannCayley Algebra in Coq and its Application to Theorem Proving in Projective Geometry, in: Automated Deduction in 
Geometry.Lecture Notes in ComputerScience, 6877, Springer Berlin Heidelberg (2011), pp 51-67.

[10].S. Amine, M. T. Masouleh, S. Caro,P. Wenger, C. Gosselin. Singularity analysis of 3T2R parallel mechanisms using Grassmann-Cayley Algebra and Grassmann geometry. Mechanism and Machine Theory, 52 ,2012), 326-340.

[11]. D. Bondyfalat. Interaction entre Symbolique et Numérique Application á la Vision Artificielle. $\mathrm{PhD}$ Thesis ,(2000)Université de Nice ,Sophia Anti Polis.

[12]. S. Caro , G. Moroz , T.Gayral ,D. Chablat, C. Chen . Singularity Analysis of a Six-Dof Parallel Manipulator Using Grassmann-Cayley Algebra and Gröbner Bases. J. Angeles et al. (Eds.) Brain, Body and Machine. Advances in Intelligent and Soft Computing,83, Springer Berlin Heidelberg (2010),pp341-352.

[13].D. Kanaan, P. Wenger ,D.Chablat. Singularity Analysis of Limited-DOF Parallel Manipulators Using GrassmannCayley Algebra. Advances in Robot Kinematics: Analysis and Design. Springer Netherlands(2008),pp 59-68.

[14].D. Kanaan, P. Wenger, S.Caro, D. Chablat. Singularity Analysis of Lower Mobility Parallel Manipulators Using Grassmann-Cayley Algebra. IEEE Transactions on Robotics ,25, no.5 (2009) 995-1004.

[15]. S. Amine, M. T. Masouleh, S. Caro , P. Wenger, C. Gosselin. Singularity Conditions of 3T1R Parallel Manipulators with Identical Limb Structures Semaan . ASME Journal of Mechanisms and Robotics ,4, 1 (2012).

[16].Hongbo Li. From Past to Future: Graßmann's Work in Context. Projective Geometric Theorem Proving with Grassmann-Cayley Algebra. Graßmann Bicentennial Conference,September 2009 Springer Basel (2011) pp 275285.

[17]. O. Faugeras,T. Papadopoulo. Grassmann-Cayley algebra for modeling systems of cameras and the algebraic equations of the manifold of trifocal .IRIA,3225,(1997).

[18]. Lennart RUBBERT .Conception de Mécanismes Compliants pour la Robotique Chirurgicale. $P h-D$ Dissertation .Université de Strasbourg.(2012),pp96.

[19]. S. Amine ,S. Caro.Singularity Conditions of LowerMobility Parallel Manipulators Based on Grassmann-Cayley Algebra. L'Agence nationale de la recherche. Retrieved December 1, 2013, from http ://www.irccyn.ecnantes.fr/ caro/SIROPA/GUIGCASiropa.jar

[20]. M-S. Tsai, T-N. Shiau, Y-J. Tsai, T-H. Chang. Direct Kinematic Analysis of a 3-PRS Parallel Mechanism. Mechanism and Machine Theory, 38, (2003)71-83.

[21]. Y.Li,Q.Xu. Kinematic Analysis of a 3-PRS Parallel Manipulators. Robotics and Computer-Integrated Manufacturing 23 (2007) 395-408.

[22]. M. Karouia, J.M.Hervé, P.Bidaud . Analyse des Singularités du Manipulateur Parallèle Sphérique Isostatique 3-RCC. 17e Congrès Français de Mécanique(2005).

[23]. Z. Niu and M. Lv. Kinematics Analysis for a NewStyle Machine Tool Based on 3-PRS Parallel Robot. C.Xiong et al. (Eds.): ICIRA 2008, Part II, LNAI ,5315,Springer-Verlag Berlin Heidelberg 2008 pp. 339-347. [24]. A.Rezaei, A. Akbarzadeh , P. M. Nia , M-R. Akbarzadeh-T. Position, Jacobian and Workspace Analysis of a 3-PSP Spatial Parallel Manipulators. Robotics and Computer-Integrated Manufacturing, 29, (2013) 158-173.
[25]. D.M. Rocha, M. M. da Silva. Workspace and Singularity Analysis of Redundantly Actuated Planar Parallel Kinematic Machines. Proceedings of the $X V$ International Symposium on Dynamic Problems of Mechanics.(2013).

[26]. R.Dadarlat, N. Plitea, B. Konya,C. Vaida, D. Pisla. Workspace and Singularities Analysis of a 6-DOF Parallel Mechanism with Two Kinematic Chains for Platform Guidance,in: F. Viadero and M. Ceccarelli (eds.), New Trends in Mechanism and Machine Science, Theory and Applications in Engineering Mechanisms and Machine Science 7, Springer Dordrecht Heidelberg New York London(2013),pp33-41.

[27]. Y. Cao , C. Gosselin , P. Ren and H. Zhou .Orientationability Analyses of a Special Class of the Stewart-Gough Parallel Manipulators using the unit Quaternion Representation. Advanced Robotics, 27, no.2,(2013) 147-158.

[28]. H-B. Choi and J. Ryu. Singularity analysis of a four degree - of - freedom Parallel Manipulator based on an expanded $6 \times 6$ Jacobian matrix. Mechanism and Machine Theory ,57, (2012) 51-61.

[29]. A. A Selvakumar., R .P. Babu, R.Sivaramakrishnan. Simulation and Singularity analysis of 3 - PRS Parallel Manipulator. Proceedings of IEEE, International Conference on Mechatronics and Automation(2012) 22032207.

[30]. S.A. Joshi, L-W. Tsai. Jacobian Analysis of LimitedDOF Parallel Manipulators. Journal of Mechanical Design, 124, ( 2002) 254- 258.

[31]. X. Ding, Y. Yang, J. S. Dai. Design and Kinematic Analysis of a Novel Prism Deployable Mechanism. Mechanism and Machine Theory ,63, (2013) 35-49.

[32].D. Zlatanov, I. A. Bonev, C. M.Gosselin. Constraint Singularities of Parallel Mechanisms. Proceedings of the IEEE. International Conference on Robotics and Automation, 1,(2002). 496-502.

[33]. A. Wolf, E. Ottaviano, M. Shoham, M. Ceccarelli. Application of line geometry and linear complex approximation to singularity analysis of the 3-DOF CaPaMan Parallel Manipulator. Mechanism and Machine Theory ,39,(2004) 75-95.

[34]. X. Cheng, Y.M. Huang, F. Gao, Y.G. Zhang ,Z.B Peng. Study on Kinematic Characteristics and Singularities of a 3-DOF Parallel Robot. Proceedings of. the Third International Conference on Machine Learning and Cybernetics, 5,(2004) 2870- 2873.

[35].J-P.Merlet. Parallel Robots -2e Edition. Chap6: Singular Configuration .Solid Mechanics and Its Applications . Springer Netherlands, (2006) pp 185.

[36].Tom Davis. Projective Geometry. Mathematical Circles Topics, (2001). Retrieved December 1, 2013, from http://www.geometer.org/mathcircles/projective.pdf [37].Lilian Aveneau.Les Coordonnées de Plücker Revisitées. Revue Électronique Francophone d'Informatique Graphique, 3, no.2, (2009)59-68.

[38].Roy Featherstone. Plücker Basis Vectors. Proceedings IEEE International Conference on Robotics and Automation, Orlando Fl, (2006) 1892-1897. 
[39].Matthew T. Mason. Mechanic of Robotic Manipulation - Intelligent Robotics and Autonomous Agents Series, Bradford Book, (2001) pp 22, 97.

[40].R. S. Ball. A Treatise on the Theorie of Screws.Cambrige University Press,(1900).

[41].B.Siciliano ,O. Khatib. Springer Handbook of Robotics. Springer-Verlag Berlin Heidelberg,( 2008)pp14-23.

[42].S. Stramigioli , H. Bruyninckx .Geometry and Screw Theory for Robotics. IEE,International Conferences on Robotics and Automation (2001), Tutorial(T9) .

[43].Lung-Wen Tsai. The Jacobian Analysis of a Parallel Manipulator Using the Theory of Reciprocal Screws. Digital Repository at University of maryLand. Institute for Systems Research Technical Report .34,(1998) Retrieved December 1, 2013, from

http://drum.lib.umd.edu/bitstream/1903/5952/1/TR_98-

34.pdf

[44].A. Wolf, M. Shoham. Screw Theory Tools for the Synthesis of the Geometry of a Parallel Robot for a given Instantaneous Task. Mechanism and Machine Theory, 41,no.6, (2006) 656-670.

[45].J. S.Dai , Ohn Rees Jones. Interrelationship between Screw Systems and corresponding Reciprocal Systems and Applications. Mechanism and machine theory, 36, no5,(2001)633-651.

[46].F. Hao and J. M. McCarthy. Conditions for line-based Singularities in Spatial Platform Manipulators. Journal of Robot. System, 15, no.1, (1998)43-55.

[47].H. Lipkin ,J. Duffy.The Elliptic Polarity of Screws. ASME Journal of Mechanism and Transmissition and Automation in Design,107, no. 3(1985)377-387.

[48].T.-S. Zhao, J. S. Dai, Z. Huang. Geometric Analysis of Overconstrained Parallel Manipulators with three and four Degrees Of Freedom, JSME ,International Journal Series C .Mech. Syst., Mach. Elements Manuf.,45, no. 3, (2002)730740.

[49].H. linkpin ,J. duffy. Analysis of Industrial Robot via Theory of Screw. IEEE, International Conference on Computer Science and Automation Engineering,3 , (2011) 40-46.

[50].X. Kong ,C. Gosselin . Springer Tract in advanced robot. Type synthesis of parallel mechanisms,33, SpringerVerlag Berlin Heidelberg (2007)pp,88-92.

[51].L. W.Tsai. Robot Analysis: The Mechanics of Serial and Parallel Manipulators. John Wiley \& Sons, New York, NY(1999).pp223-258.

[52].Neil L White. Multilinear Cayley Factorization. Journal of Symbolic Computation, 11, no.5-6, (1991), 421438.

[53].T. Mc Millan ,Neil White .The Dotted Straightening Algorithm. Journal of Symbolic Computation, 11, no.5-6, (1991)471-482.

[54].B. Sturmfels, W. Whiteley .On The Synthetic Factorization of Protectively Invariant Polynomials. Journal of Symbolic Computation, 11, no.5-6, (1991),439-453.

[55].H. Li and Y. Wu. Automated Short Proof Generation for Projective Geometric Theorems with Cayley and Bracket Algebra. I. Incidence Geometry. Journal of Symbolic Computation 36 ,2003) 717-762.
[56].J. A. Carretero, R. P. Podhorodeski, M. A. Nahon, C. M. Gosselin. Kinematic Analysis and Optimization of a New Three Degree-of-Freedom Spatial Parallel Manipulator. Journal of Mechanical Design,122,(2000)1724.

[57].G. Pond, J.A. Carretero. Kinematic Analysis and Workspace determination of the Inclined PRS Parallel Manipulator, in: Proceedings of RoManSy 2004: 15th CISM-IFToMM Symposium on Robot Design, Montreal, Canada (2004).

[58]. G. Pond, J.A. Carretero. Architecture Optimization of 3-PRS variants for Parallel Kinematics Machining .Robotics and Computer-Integrated Manufacturing, 25, (2009)64-72 . [59].G. Csurka, O.D. Faugeras.Computing ThreeDimensional Projective Invariants from a Pair Of Images using the Grassmann-Cayley Algebra .Image and Vision Computing,(1995)150-157.

[60].S.HUOT, C.Colin.MARINA : un outil de reconstruction de bâtiments en $3 \mathrm{D}$ à partir d'images.Mémoire de DEA, (2000) Universitécole Nationale Supérieure des Techniques Industrielles et des Mines de Nantes

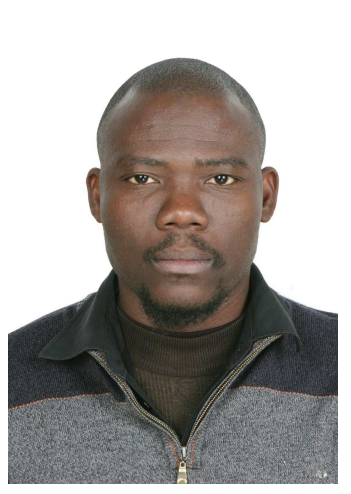

\section{BIOGRAPHY}

AKONDE L. D. Clément. Currently $\mathrm{PhD}$ Student in Mechatronics at School of Mechanical Science and Engineering .Huazhong University of Science and Technology (Wuhan-China).He received Master in Mechatronics at School of Mechanical Science and Engineering of Huazhong University of Science and Technology. Research fields: Mechanic of Robot and Mechanism of Robot Manipulators .Mathematics of Robot Manipulation 